\title{
High Spectrum Narrowing Tolerant 112 Gb/s Dual Polarization QPSK Optical Communication Systems Using Digital Adaptive Channel Estimation
}

Zhang, Xu; Pang, Xiaodan; Dogadaev, Anton Konstantinovich; Tafur Monroy, Idelfonso ; Zibar, Darko; Younce, Richard

Published in:

OFC/NFOEC Technical Digest

Publication date:

2012

Document Version

Publisher's PDF, also known as Version of record

Link back to DTU Orbit

Citation (APA):

Zhang, X., Pang, X., Dogadaev, A. K., Tafur Monroy, I., Zibar, D., \& Younce, R. (2012). High Spectrum Narrowing Tolerant $112 \mathrm{~Gb} / \mathrm{s}$ Dual Polarization QPSK Optical Communication Systems Using Digital Adaptive Channel Estimation. In OFC/NFOEC Technical Digest (pp. JW2A.49). Optical Society of America.

\section{General rights}

Copyright and moral rights for the publications made accessible in the public portal are retained by the authors and/or other copyright owners and it is a condition of accessing publications that users recognise and abide by the legal requirements associated with these rights.

- Users may download and print one copy of any publication from the public portal for the purpose of private study or research.

- You may not further distribute the material or use it for any profit-making activity or commercial gain

- You may freely distribute the URL identifying the publication in the public portal 


\title{
High Spectrum Narrowing Tolerant 112 Gb/s Dual Polarization QPSK Optical Communication Systems using Digital Adaptive Channel Estimation
}

\author{
Xu Zhang ${ }^{(1)}$, Xiaodan Pang ${ }^{(1)}$, Anton Dogadaev ${ }^{(1)}$, Idelfonso T. Monroy ${ }^{(1)}$, Darko Zibar ${ }^{(1)}$, Richard Younce $^{(2)}$ \\ (1) Department of Photonic Engineering, Technical University of Denmark, Ørsteds Plads DK2800, Kgs, Lyngby, Denmark \\ (2) Tellabs, 1415 West Diehl Road, Naperville, IL 60563 USA. \\ xuzhn@fotonik.dtu.dk
}

\begin{abstract}
We experimentally demonstrate high spectrum narrowing tolerant 112-Gb/s QPSK polarization multiplex system based on digital adaptive channel estimation method. The proposed algorithm is able to detect severe spectrum-narrowed signal even with $20 \mathrm{GHz} 3 \mathrm{~dB}$ bandwidth. OCIS codes: (060.1660 Coherent communications)
\end{abstract}

\section{Introduction}

With the rapid growth of capacity requirements of optical transmission networks, there is a strong need to realize high capacity and high spectral efficient (SE) optical communication systems. The ultra-dense wavelength diversity multiplexing (U-DWDM) technology based on advanced modulation format is a promising solution and is being intensively studied [1,2]. U-DWDM technology requires optical or electrical pre-filtering to fulfil Nyquist sampling criterion [3]. Meanwhile, to facilitate ease of optical networking, tolerance of spectrum narrowing is critical, as signal channel spacing is degraded after transmission through optical channel, especially when reconfigurable optical add/drop multiplexers (ROADMs) nodes are used in the network [4]. Spectrum narrowing severely induce inter symbol interference (ISI) between continual transmitted sequence. Therefore, ISI compensation is essential to improve U-DWDM system performance in terms of optical signal to noise ratio (OSNR). Conventional digital signal processing (DSP) algorithm using adaptive decision feed forward equalizer is an inefficient solution, since this finite impulse response (FIR) filter enhance noise during compensation of spectrum narrowing [5]. Although maximum-likelihood sequence estimation (MLSE) method has been successfully proved to mitigate ISI [6], the complexity of MLSE is exponentially increasing with the length of transmitted sequence. When the sequence length is large, optimal MLSE becomes unfeasible. Therefore, in order to achieve high spectrum narrowing tolerant optical communication system, we implement adaptive channel estimation DSP algorithms combined with decision feedback equalizer. As a result, we experimentally demonstrate the high spectrum narrowing tolerance of proposed algorithms for $112 \mathrm{~Gb} / \mathrm{s}$ dual polarization (DP) quadrature phase shift keying (QPSK) transmission systems.

\section{Experiment Setup}

Figure 1(a) shows the experiment setup of $112 \mathrm{~Gb} / \mathrm{s}$ DP QPSK optical communication system. A DFB laser operating at $1549.53 \mathrm{~nm}(193.47 \mathrm{THz})$ with $10 \mathrm{MHz}$ line-width is used as optical signal source to generate the QPSK signal. The pulse pattern generator (PPG) working at $28 \mathrm{Gbaud} / \mathrm{s}$ is operated as electrical signal source with $2^{15}-1$ bit length to drive the optical QPSK modulator. After the QPSK modulator, optical channel is separated into two orthogonal polarizations by a polarization beam splitter (PBS). Afterward, two branches of orthogonally polarized optical channels are combined by a polarization beam combiner (PBC) to generate the $112 \mathrm{~Gb} / \mathrm{s}$ DP QPSK signal. The Finisar ${ }^{\circledR}$ wave-shaper is then used as spectrum pre-filtering optical bandpass filter (OBPF). The OBPF is able to tune full width at half maximum (FWHM) of transmitted signal. FWHM is defined as 3dB bandwidth of transmitted signal. At the receiver side, a pre-amplified coherent receiver structure is implemented. A tunable optical band-pass filter (with $0.5 \mathrm{~nm} / 62.5 \mathrm{GHz}$ FWHM) is used after the pre-amplifier in order to remove ASE noise from the EDFA. The local oscillator (LO) is a wavelength tunable external cavity laser (ECL) with $100 \mathrm{kHz}$ line-width. In the coherent receiver structure, tuning LO wavelength enables channel selection due to optical heterodyning at the photodiode. The coherent receiver consists of two $90^{\circ}$ hybrids and balanced detectors. The $80 \mathrm{~Gb} / \mathrm{s}$ digital sampling oscilloscope at the coherent receiver is used to sample the inphase and quadrature components. Figure 1(b) shows the back to back transmission bit error rate (BER) performance in terms of OSNR. In both results of simulation and experiment for back to back transmission, at $20 \mathrm{~dB}$ OSNR measurement point, BER performances are approaching zero, and at $16 \mathrm{~dB}$ OSNR measurement point, BER performances are around $10^{-3}$. In the experiment, we focused on these two OSNR measurement points (OSNR=16dB and OSNR=20dB) and use DSP algorithm to compensate ISI induced by spectrum narrowing effect. We also employed constant modulus algorithm (CMA) to enable polarization rotation compensation, frequency and phase off set removal, QPSK demodulation and bit error detection. 


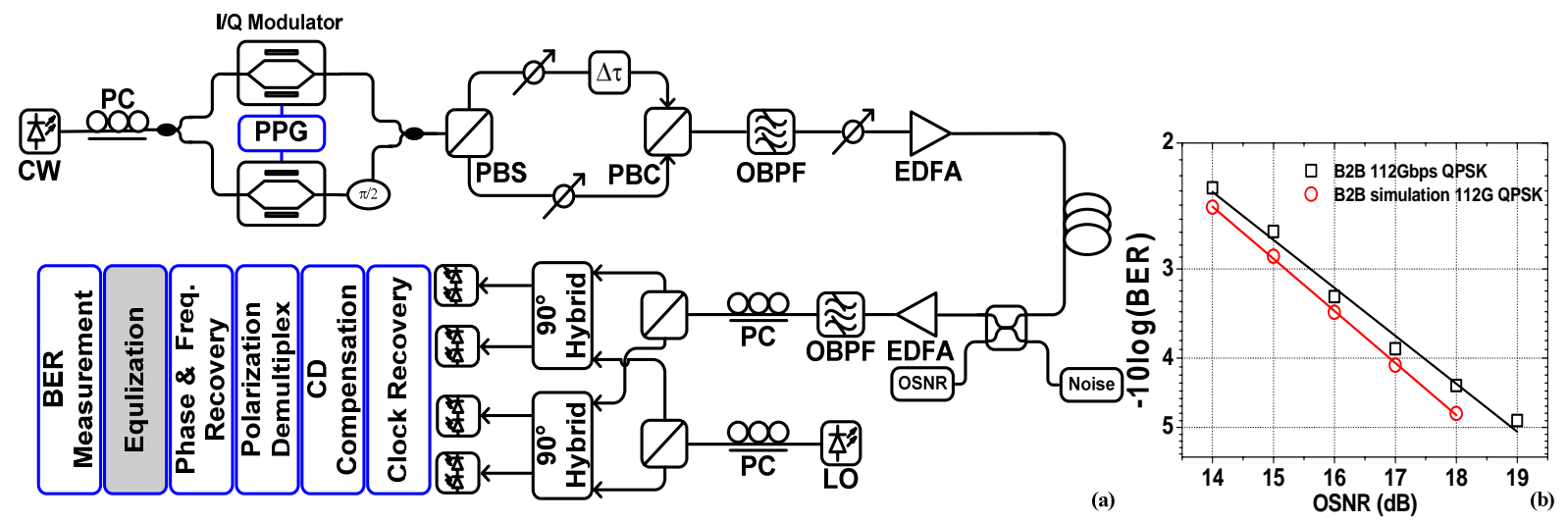

Figure. 1. (a) Experiment setup. (b) back to back BER vs. OSNR performance.

\section{DSP Algorithms and Experiment Results}

The proposed DSP algorithm schematic is shown in Figure 2. The polarization multiplexed signals are firstly fed into coherent detection receiver. Afterward, the received signals are sampled by analog to digital convertor (ADC) to 2 samples per symbol. The output digitized signals are fed into a butterfly filter composed of four FIR filters. CMA algorithm is used to improve polarization demultiplex performance and compensate polarization rotation. The signals are sent to carrier recovery block to demultiplex inphase and quadrature components and then to the adaptive channel estimation block. The adaptive channel estimation requires estimation of noise level $\eta_{k}$; feed forward FIR tap coefficients $c_{k}$ and feed back equalizer tap coefficients $p_{k}$. For instance, in one orthogonal polarization, denoted as $x$-polarization, input of channel estimation is $x(k)$. Since a finite length feed forward equalizer is unable to cancel noncausal ISI completely, the feed back filter coefficients should be optimized according to the MMSE criterion whereby the additive noise power resulting from residual ISI is minimized. The correct channel estimation of $c_{k}$ and $p_{k}$ is able to minimize minimum mean square error (MMSE) between received signals and decision signals [7], i.e. $\min \left\{E\left[x^{\prime}(k)-\eta-x^{\sim}(k)\right]^{2}\right\}$, where $x^{\prime}(k)=c_{k} x(k)$ and $x^{\prime \prime}(k)=x^{\prime}(k)-p_{k} x^{\sim}(k)$ and $\tilde{x}^{\sim}(k)$ is the slicer output from $x^{\prime \prime}(k)$. The stochastic adaptive algorithm to find $c_{k}, \eta_{k}$ and $p_{k}$ can be implemented using the LMS algorithm [8].

We denote error factor as $e_{k}=x^{\prime}(k)-\eta_{k}-x^{\sim}(k)$. Then we have following update equations: $c_{k+1}=c_{k}+\mu e_{k} x(k)$; $p_{k+1}=p_{k}-\gamma e_{k} x^{\sim}(k)$ and $\eta_{k+1}=(1-\rho) \eta_{k}+\rho e_{k}$. Here, $\mu$ is the step size of feed forward FIR equalizer; $\gamma$ is the step size of feed back equalizer and $\rho$ is an integration factor (a small value used to average $e_{k}$ over time $k$ ) [8]. As a result, symbol estimation $x_{m}{ }^{\sim}(k)$ is a estimation vector: $\{x(k-n), x(k-n-1), x(k-n-2) \ldots x(k-1)\}$, where $n$ is the length of feed back equalizer. Operation of subtracting $x_{m}{ }^{\sim}(k)$ combined with proper channel estimation coefficients from received signals is used to cancel most of precursor ISI [9]. According to accuracy estimation requirement of noise level, length of feed back equalizer can not be insufficient. Compared to the complexity requirement of MLSE algorithm, our proposed adaptive channel estimation algorithms are practical.

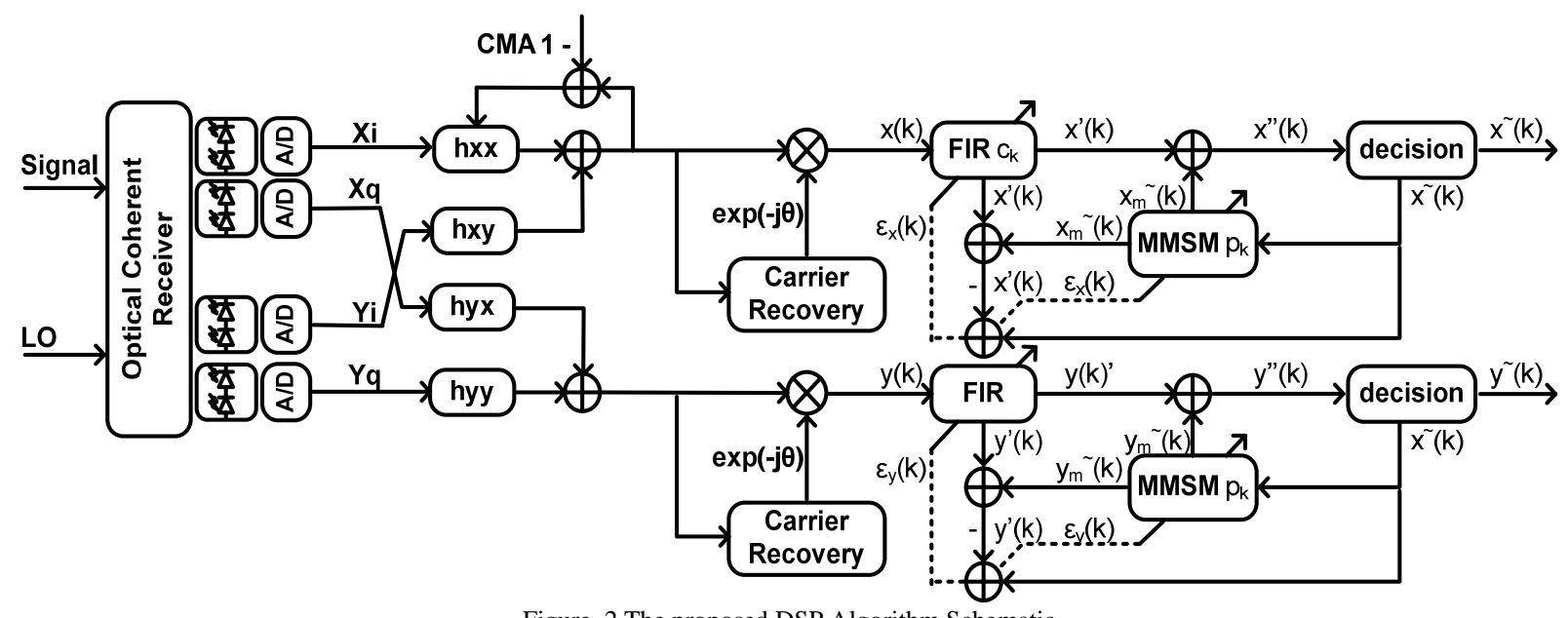

Figure. 2 The proposed DSP Algorithm Schematic 


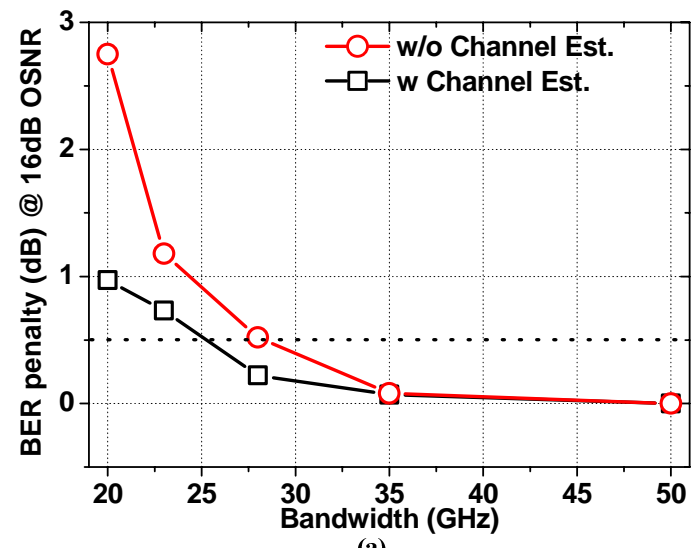

(a)

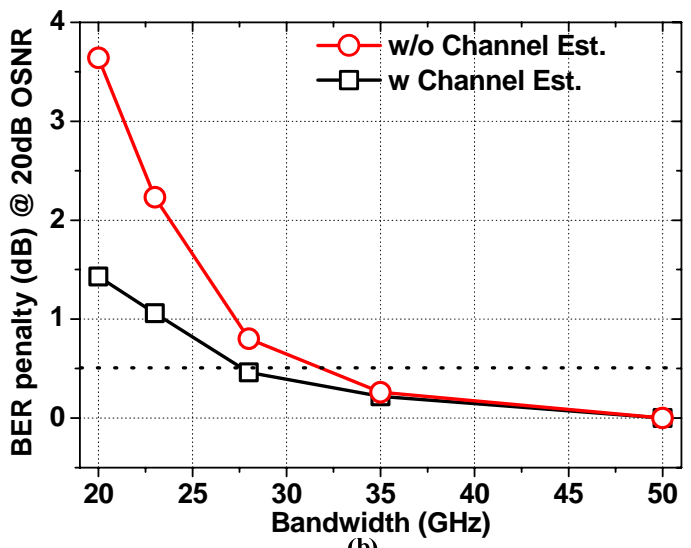

(b)

Figure. 3 BER penalty vs. bandwidth narrowing with and without adaptive channel estimation algorithm. (a) @ 16dB OSNR. (b) @ 20dB OSNR

Figure 3 shows experiment results. In our experiment, we focus on $20 \mathrm{~dB}$ OSNR and $16 \mathrm{~dB}$ OSNR measurement points. At $20 \mathrm{~dB}$ OSNR, in case of without spectrum narrowing effect, BER performance is error free. As the signal spectrum space degrading, BER penalty grow dramatically. Meanwhile, using adaptive channel estimation algorithm, strongly reduce the BER penalty. Especially at $20 \mathrm{GHz}$ bandwidth (71\% of original signal bandwidth), BER penalty is reduced $2 \mathrm{~dB}$ by using adaptive channel estimation algorithms. At $20 \mathrm{~dB}$ OSNR, in case of with out spectrum narrowing effect, BER performance is around $10^{-3}$. At $20 \mathrm{GHz}$ bandwidth (71\% of original signal bandwidth), BER penalty is also reduced $2 \mathrm{~dB}$ by using adaptive channel estimation algorithms.

\section{Conclusion}

By using our proposed adaptive channel estimation DSP algorithms, we successfully demonstrate high spectrum narrowing tolerant 112-Gb/s QPSK polarization multiplex optical communication systems. The experiment results show that in case of 71\% spectrum narrowing, our proposed algorithms mitigate $2 \mathrm{~dB}$ BER penalties with both 16 $\mathrm{dB}$ OSNR and $20 \mathrm{~dB}$ OSNR cases. Compared to high computational complexity requirement of MLSE algorithm, our proposed adaptive channel estimation DSP algorithm is feasible and practical.

\section{References}

[1] Nakazawa, M., Okamoto, S., Omiya, T., Kasai, K., and Yoshida, M. "256-QAM (64Gb/s) coherent optical transmission over 160 km with an optical bandwith of 5.4 GHz” IEEE Photonic Technol. Lett., 2010, vol.22, no.3.

[2] Valeria Arlunno, Xu Zhang, Knud J.Larsen, Darko Zibar and Idelfonso Tafur Monroy "Digital Non-Linear Equalization for Flexible Capacity Ultradense WDM Channels for Metro Core Networking” ECOC 2011 Tu3.K6.

[3] T. Sugihara, T. Kobayashi, Y. Konishi, S. Hirano, K. Tsutsumi, K. Yamagishi, T. Ichikawa, S. Inoue, K. Kubo, Y. Takahashi, K. Goto, T. Fujimori, K. Uto, T. Yoshida, K. Sawada, S. Kametani, H. Bessho, T. Inoue, K. Koguchi, K. Shimizu, and T. Mizuochi, “43 Gb/s DQPSK Preequalization employing 6-bit, 43GS/s DAC Integrated LSI for Cascaded ROADM Filtering” OFC 2010 PDPB6.

[4] S. Gringeri, R. Egorov, B. Basch, and G. Wellbrock, "Real-Time 127-Gb/s Coherent PM-QPSK Transmission over 1000km NDSF with >10 Cascaded 50GHz ROADMs” ECOC 2010 P4.09.

[5] S. J. Savory “Digital filters for coherent optical receivers,” Opt. Express 16, 804-817 (2008).

[6] K. Horikoshi, E. Yamazaki, T. Kobayashi, E. Yoshida, and Y. Miyamoto, "Spectrum-narrowing tolerant signal-processing algorithm using maximum-likelihood sequence estimation for coherent optical detection” Electronics Letter 12 ${ }^{\text {th }}$ May 2011, vol.47, no.10.

[7] Tommaso Foggi, Giulio Colavolpe, Enrico Forestieri, and Giancarlo Prati, "Channel Estimation Algorithms for MLSD in Optical Communication Systems” IEEE Photonic Technol. Lett., October 2006, vol.18, no.19.

[8] Wonzoo Chung, “Channel Estimation Methods Based on Volterra Kernels for MLSD in Optical Communication Systems” IEEE Photonic Technol. Lett., February 2010, vol.22, no.4.

[9] Heinrich Meyr, Marc Moeneclaey, and Stefan A. Fechtel Digital Communication Receiver-synchronization, channel estimation, and signal processing JOHN WILEY \& SONS, INC. 